\title{
Intractable Epistaxis Secondary to Traumatic Pseudoaneurysm of Internal Carotid Artery
}

\author{
Nikitha Pillai ${ }^{1}$, Smitha Soubhagya Gangaraj ${ }^{2}$
}

\begin{abstract}
Aim and objective: A rare presentation of intractable massive recurrent epistaxis diagnosed and managed.

Background:Traumatic intracranial pesudoaneurysm is a rare complication of blunt trauma. Intracranial internal carotid artery pseudoaneurysm may rupture leading to delayed onset, massive, recurrent epistaxis, which requires an emergency intervention.

Case description: Here, we present a case of recurrent massive intractable epistaxis due to a sphenoidal prolapse of supraclinoid part of right internal carotid artery, presenting as a pseudoaneurysm, secondary to a post-traumatic fracture dehiscence of roof of sphenoid, diagnosed with the help of a computed tomography angiography. The patient was treated successfully with a primary endovascular coiling.

Conclusion: Intractable epistaxis due to a post-traumatic pseudoaneurysm is quite rare and can be fatal and hence need to be considered in the differential diagnosis of massive recurrent uncontrollable epistaxis and treated at the earliest.

Clinical significance: The reach of presentations of such rare cases should be broadened as these are difficult to be diagnosed and managed. Inadequate and untimely diagnosis and management of such cases can cost the life of the patients.

Keywords: Craniofacial trauma, Endovascular coiling, Intractable epistaxis, Pseudoaneurysm, Recurrent.

Clinical Rhinology An International Journal (2019): 10.5005/jp-journals-10013-1362
\end{abstract}

\section{BACKGROUND}

Epistaxis is a very common medical condition, mostly self-limiting. Around $7-60 \%$ of population have epistaxis at sometime or other of their lives. ${ }^{1}$

The various causes of epistaxis include dryness of nasal cavity, digital trauma, the use of NSAIDs and anticoagulants, alcohol consumption, septal deviation, hypertension, and blood dyscrasias, all of which may be managed conservatively and/or with nasal packing, either anterior or posterior, or with local measures such as applying pressure to nostrils, chemical or electrical cauterization, and by treating any underlying causes.

Recurrent intractable epistaxis following a blunt craniofacial trauma can be due to a traumatic pseudoaneurysm of the internal carotid artery (ICA). It is a rare condition with an incidence reported of $1.1 \% .^{2}$

The most common manifestation of traumatic pseudoaneurysm is a delayed onset recurrent intractable epistaxis. ${ }^{3}$ It demands prompt recognition as it can be fatal if not managed at the earliest. The treatment of choice here mainly involves endovascular intervention.

\section{Case Description}

In December 2017, a 20-year-old male patient had visited with complaints of transiently recurrent and profuse epistaxis from both sides of the nose, which started 2 weeks following fall from a height. Each episode of bleeding would be present for a duration of 15 minutes to half an hour with a loss of about $400-500 \mathrm{~mL}$ of blood and would subside on its own spontaneously or with pressure or medications (Fig. 1A).

On examination, patient was anemic with a hemoglobin of $6 \mathrm{~g} /$ $\mathrm{dL}$. All the bleeding and clotting parameters were normal. Anterior rhinoscopic examination of nose showed a deviated nasal septum to the left. Diagnostic nasal endoscopy done confirmed our findings
1,2Department of ENT, Kempegowda Institute of Medical Sciences, Bengaluru, Karnataka, India

Corresponding Author: Nikitha Pillai, Department of ENT, Kempegowda Institute of Medical Sciences, Bengaluru, Karnataka, India, Phone: +91 9591155315, e-mail: pillai_nikitha@hotmail.com

How to cite this article: Pillai N, Soubhagya Gangaraj S. Intractable Epistaxis Secondary to Traumatic Pseudoaneurysm of Internal Carotid Artery. Clin Rhinol An Int J 2019;12(2 and 3):63-65.

Source of support: Nil

Conflict of interest: None

and also confirmed the absence of any bleeding points and mass in both the nasal cavities and in the nasopharynx.

X-ray paranasal sinuses and X-ray nasopharynx were also done to rule out the presence of any mass. Computed tomography (CT) brain done following the incident showed a fracture of the right sphenoid greater wing and lateral pterygoid plate with right sphenoid hemosinus. Magnetic resonance image (MRI) of brain was done and reported normal. CT-PNS done showed sphenoid sinusitis with bilateral hypertrophied inferior turbinates and deviated nasal septum to the left. MRI-PNS also was in consistent with the CT report showing sphenoid sinusitis.

Having ruled out all possible systemic causes, epistaxis and the resultant anemia was thought to be due to a local cause, for which septoplasty was performed in January 2018, after having given multiple blood transfusions to correct the anemia. The patient was discharged and followed up.

After around 4 weeks, patient returned with the same complaints of recurrent episodic epistaxis, and at this time, he was advised to get a CT angiogram done. It showed posttraumatic fracture dehiscence of superolateral part of roof of right sphenoid sinus with associated intrasphenoidal prolapse 


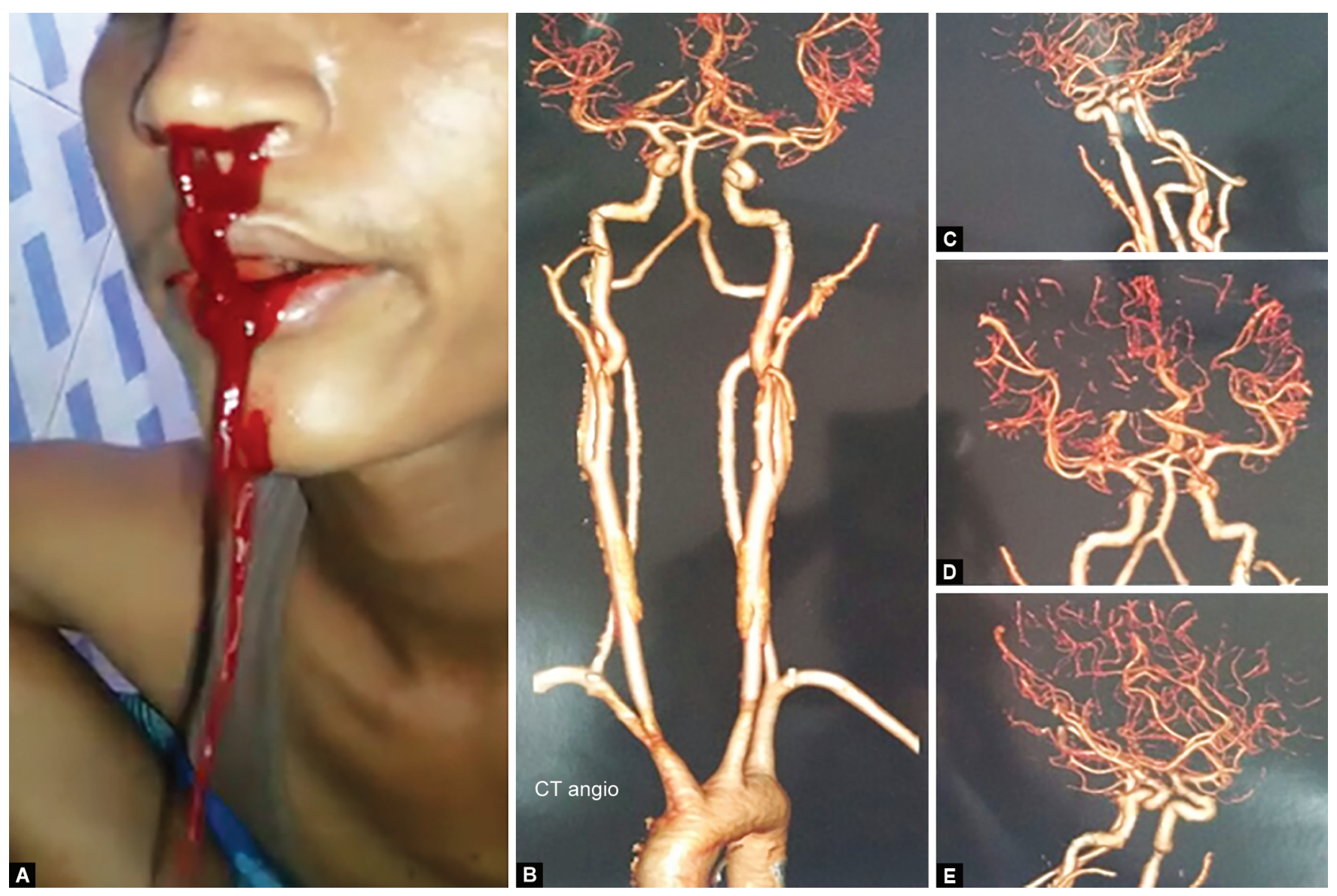

Figs $1 \mathrm{~A}$ to $\mathrm{E}$ : (A) Patient presenting with profuse intractable epistaxis; (B to E) CT angio showing extra- and intracranial vasculatures

of supraclinoid part of right ICA with surrounding mucosal thickening/clot (Figs 1B to E).

The patient was referred to a higher center for neuroradiological intervention. A check angiogram was performed and a right carotid artery cavernous segment psuedoaneurysm of $6.5 \times 5.3 \mathrm{~mm}$ was noted projecting into right sphenoid sinus with good cross-flow.

In March 2018, patient underwent coiling and occlusion of the psuedoaneurysm of cavernous segment of the right ICA at its neck. Eight coils were deployed through echelon microcatheter with the help of balloon, just between the origin of ophthalmic artery and distal cavernous ICA covering the neck of the pseudoaneurysm.

Postprocedure-check angiogram showed good occlusion of the right ICA with very faint contrast filling of cervical and petrous ICA. Injection of the left ICA showed optimal cross-circulation across the anterior communicating artery to contralateral side without any venous lag. Injection of left vertebral artery showed optimal flow across the right posterior communicating with antegrade filling of ophthalmic artery and faint filling of pseudoaneurysm.

Patient was discharged and has been on regular follow-up with no further complaints.

\section{Discussion}

Epistaxis is one of the most common medical emergencies encountered by otorhinolaryngologists. It is usually caused due to a disruption of the external carotid artery branches, especially the internal maxillary artery. ${ }^{4}$ Trauma to the skull base segments can injure the intracranial ICA leading to a delayed onset recurrent and massive epistaxis. ${ }^{5}$

Injury to a blood vessel can weaken its wall leading to an abnormal widening or ballooning of a portion of the vessel, called aneurysm. A true aneurysm involves all three layers of the vessel. A false or pseudoaneurysm due to trauma involves partial vessel transection and formation of a hematoma and presents as an outpouching. $^{6}$

The counter pressure of the hematoma stops the bleeding temporarily. The circulating blood continues to fill into the unclotted portion of the hematoma around the injured vessel wall, in continuity of the arterial lumen. The hematoma liquefies in around 1-3 weeks and may rebleed. There is formation of a fibrous capsular wall and development of an epithelial lining in response to the inflammatory reaction created by the blood in the surrounding tissue. The fibrous wall can break down due to its enlargement and weakening as a result of the continuous pulsatile forces. This can cause the repeated epistaxis. ${ }^{7-9}$

The ICA aneurysms with sphenoid extension and revealed by epistaxis are rare. Aneurysms that involve cavernous segment of ICA account for $3-5 \%$ of all intracranial aneurysms. ${ }^{10}$ Most of these aneurysms occur following trauma, but postoperative and nontraumatic aneurysms have also been documented..$^{11}$ The cavernous ICA has an intimate relationship with sphenoid sinus, bulging into lateral sinus wall. The thin interface of tissue between these structures makes this a site for potential injury in trauma. ${ }^{12}$ 
The epistaxis caused due to this traumatic pseudoaneurysm is usually of a delayed onset but is usually recurrent and massive. It is difficult to control and can be potentially fatal by causing hemorrhagic shock or asphyxia. Mortality rate of a ruptured pseudoaneurysm is $30-50 \% .^{13}$

Conventional CT angiography is the gold standard in evaluating patients with traumatic pseudoaneurysm of ICA. ${ }^{14}$ If CT angiography and digital subtraction angiography fail to show the site of injury, and the patient continues to have epistaxis, repeat imaging is advised in 3 weeks, as $90 \%$ of patients present in 3 weeks after the injury. ${ }^{15}$

The various surgical options of treatment of pseudoaneurysm include direct clipping, wrapping, trapping, and carotid artery ligation. ${ }^{16}$ Traumatic psedoaneurysms near the skull base are hazardous to approach and challenging to repair. Hence, endovascular techniques, including detachable balloons and coils, are preferred over open surgical techniques for parent vessel occlusion and preservation of the artery and also proves advantageous by sparing the patient the need of antiplatelet therapy postprocedure, in case a stent is deployed. ${ }^{17-19}$

Coiling of the pseudoaneurysm is preferred in most cases where the neck of the pseudoaneurysm is narrow. If the neck is wide, coiling can be performed with balloon remodeling. If the vessel wall is dysplastic and neck is wide, treatment can be performed with the stent assistance prior to or after replacement of coils to reconstruct the vessel and contain the coil mass. ${ }^{19}$

\section{Conclusion}

Craniofacial trauma leading to pseudoaneurysm is a rare complication requiring emergency intervention as it can be fatal. Hence, a delayed onset recurrent massive epistaxis should alert the physician. CT angiography is the preferred diagnostic investigation. This condition can be effectively treated with endovascular coiling, as was our patient who had an ICA pseudoaneurysm post-traumatic sphenoid fracture.

\section{Clinical Significance}

This being a rare case needs to be discussed about vicariously as this is a fatal condition if not diagnosed and managed adequately and timely.

\section{ACKNOWLedgments}

The authors extend their gratitude to all the faculty members of the department of ENT, KIMS, and all the other supporting departments, and the management for giving their valuable opinions and suggestions during discussion of the case and also for extending their help and work towards this study. The late, $\mathrm{Dr}$ Anil HT, ex-Head, Department of ENT, KIMS, is being remembered for his selfless guidance all throughout.

They also extend their gratefulness to the patient and all his family members for their cooperation and patience during the course of recovery.

A special mention goes to beloved staff including Dr Jagannath, Dr KG Somashekara, Dr M Nirmala, and Dr Lasya N Raj, and all the postgraduates and interns for all their support in making this article a success.

\section{References}

1. Monux A, Tomás $M$, Kaiser $C$, et al. Conservative management of epistaxis. J Laryngol Otol 1990;104(11):868-870. DOI: 10.1017/ S0022215100114203.
2. Redekop G, Marotta T, Weill A. Treatment of traumatic aneurysms and arteriovenous fistulas of the skull base by using endovascular stents. J Neurosurg 2001;95(3):412. DOI: 10.3171/jns.2001.95.3.0412.

3. Goleas J, Paige ML, Mikhael MA, et al. Intracavenous carotid artery aneurysm presenting as recurrent epistaxis. Ann Otol Rhinol Laryngol 1991;100(7):577-579. DOI: 10.1177/000348949110000711.

4. Zhang C, Xie X, You C, et al. Endovascular treatment of traumatic pseudoaneurysm presenting as intractable epistaxis. Korean J Radiol 2010;11(6):603-611. DOI: 10.3348/kjr.2010.11.6.603.

5. Cheong JH, Kim JM, Kim CH. Bony protuberances on the anterior and posterior clinoid processes lead to traumatic internal carotid artery aneurysm following craniofacial injury. J Korean Neurosurg Soc 2011;49(1):49-52. DOI: 10.3340/jkns.2011.49.1.49.

6. Luo CB, Teng MM, Chang FC, et al. Role of CT and endovascular embolization in managing pseudoaneurysms of the internal maxillary artery. J Chin Med Assoc 2006;69(7):310-316. DOI: 10.1016/ S1726-4901(09)70264-3.

7. Celil G, Engin D, Orhan G, et al. Intractable epistaxis related to cavernous carotid artery pseudoaneurysm: treatment of a case with covered stent. Auris Nasus Larynx 2004;31(3):275-278. DOI: 10.1016/j. anl.2004.03.007.

8. Bavinzski G, Killer M, Knosp E, et al. False aneurysm of the intracavemous carotid artery. Report of 7 cases. Acta Neurochir 1997;139(1):37-43. DOI: 10.1007/BF01850866.

9. Germiller JA,Myers LL, Harris MO, etal.Pseudoaneurysm of the proximal facial artery presenting as oropharyngeal hemorrhage. Head Neck 2001;23(3):259-263. DOI: 10.1002/1097-0347(200103)23:3<259::AIDHED1027>3.0.CO;2-4.

10. Li MH, Li YD, Gao BL, et al. A new covered stent designed for intracranial vasculature: application in the management of pseudoaneurysms of the cranial internal carotid artery. AJNR Am J Neuroradiol 2007;28(8):1579-1585. DOI: 10.3174/ajnr.A0668.

11. Kumar D, Sahu GR, Kumar A, et al. Traumatic pseudoaneurysm of internal carotid artery presenting as intractable epistaxis in a case of maxillary fracture. J Postgrad Med Edu Res 2014;48(2):100-102. DOI: 10.5005/jp-journals-10028-1110.

12. Adeel $M$, Ikram M. Post-traumatic pseudoaneurysm of internal carotid artery: a caus of intractable epistaxis. BMJ Case Rep 2012;2012 10.1136/ bcr.02.2012.5927.

13. Purgina B, Milroy CM. Fatal traumatic aneurysm of the posterior inferior cerebellar artery with delayed rupture. Forensic Sci Int 2015;247:el-e5. DOI: 10.1016/j.forsciint.2014.11.003.

14. Chen D, Concus AP, Halbach VV, et al. Epistaxis originating from traumatic pseudoaneurysm of the internal carotid artery: diagnosis and endovascular therapy. Laryngoscope 1998;108(3):326-331. DOI: 10.1097/00005537-199803000-00004.

15. Fontela PS, Tampieri D, Atkinson JD, et al. Posttraumatic pseudoaneurysm of the intracavernous internal carotid artery presenting with massive epistaxis. Pediatr Crit Care Med 2006;7(3):260-262. DOI: 10.1097/01.PCC.0000216418. 01278.5E.

16. Kadyrov NA, Friedman JA, Nichols DA, et al. Endovascular treatment of an internal carotid artery pseudoaneurysm following transsphenoidal surgery. Case report. J Neurosurg 2002;96(3):624-627. DOI: 10.3171/ jns.2002.96.3.0624.

17. Bell RS, Vo AH, Roberts $\mathrm{R}$, et al. Wartime traumatic aneurysms: acute presentation, diagnosis and multimodal treatment of 64 craniocervical arterial injuries. Neurosurgery 2010;66(1):66-79. DOI: 10.1227/01.NEU.0000361285.50218.A8discussion 79.

18. Luo CB, Teng MM, Chang PC, et al. Endovascular management of the traumatic cerebral aneurysms associated with traumatic carotid cavernous fistulas. AJNH Am J Neuroradiol 2004;25:501-505.

19. Ruiz-Juretschke F, Castro E, Mateo Sierra O, et al. . Massive epistaxis resulting from an intracavernous internal carotid artery traumatic pseudoaneurysm: complete resolution with overlapping uncovered stents. Acta Neurochir (Wien) 2009;151(12):1681-1684. DOI: 10.1007/ s00701-009-0294-5. 